anaemia, transient abnormal myelopoiesis, post-transplant lymphoproliferative disorder and Fanconi anaemia. Of the remaining 154 patients diagnosed with cancer, 88 (57.1\%) were classified as 'green' according to the Royal Marsden Hospital 'Stop-light' system. 48 (31.2\%) were classified as 'orange' and $18(11.7 \%)$ were classified as red, qualifying for automatic referral to clinical genetics.

Conclusions A significant proportion of patients diagnosed with cancer in OLCHC may benefit from referral to clinical genetics and screening for underlying cancer predisposition syndromes.

\section{P490 INCIDENCE AND 5 YEARS SURVIVAL RATES OF CHILDHOOD CANCER DIAGNOSED LESS THAN 1 YEAR OLD IN IRELAND 2007-2017}

Jsun Loong Wong*, Frieda Clinton, Maria Carroll, Andrea Malone, Aengus O'Marcaigh, Owen Patrick Smith, Jane Pears, Michael Capra, Cormac Owens. Our Lady's Children's Hospital, Dublin, Ireland

\subsection{6/archdischild-2019-epa.826}

Introduction Childhood cancer is the second commonest cause of death in children in developed countries. Childhood cancer survival rates has improved over the last decade with the advancement of diagnostic procedures and continuous improvement of multimodal treatment strategies. According to the National Cancer Registry of Ireland, an average of 137 cancers were diagnosed per year in children under the age of 15 between 1994 and 2014. The 5 -year overall survival rate for this entire cohort was $81 \%$. It is well documented that survival rates in paediatric oncology vary depending on specific cancer diagnosis, age of the patient at diagnosis and disease stage.

Aim We describe the incidence of cancer in very young children and the influence of age on outcome in children diagnosed with cancer in Ireland under the age of 1 between 2007-2017.

Method Data were extracted from the database of the National Children's Cancer Service (NCCS) based at Our Lady's Children's Hospital, Dublin. The data presented refer to the International Classification of Childhood Cancer (ICCC) version 3 with the inclusion of Langerhans Cell Histiocytosis (LCH).

Result 185 patients were diagnosed with paediatric cancer under the age of 1 at the time of their initial diagnosis. 159 $(85.5 \%)$ patients were diagnosed with solid tumours or LCH. $86(46.5 \%)$ were male. The average age at diagnosis was 5.24 (range 0-12) months. 19 (10.3\%) patients were diagnosed following an abnormal antenatal scan. The most common cancers diagnosed were neuroblastoma (22.7\%), CNS tumours (19.5\%) and leukaemia (13.5\%).

$158(85.5 \%)$ patients received treatment. $124(78.4 \%)$ patients received chemotherapy as part of their treatment. 20 $(12.7 \%)$ patients received radiotherapy. The mean age for radiotherapy was 1.35 years (range 0.17-4). 18 (9.7\%) patients received stem cell transplant/rescue. The cumulative overall survival rate at 5 years is $80 \%$. CNS tumours have the worst prognosis followed by leukaemias. 35 (18.9\%) patients have relapsed during follow up, and 20 are alive in follow-up. Conclusion Incidence rates of specific paediatric cancer types vary according to the age at diagnosis. There is no difference in survival rates between children diagnosed with cancer under 1 year of age and older children. Outcomes at the NCCS compare favourably with international standards.
${ }^{1}$ Thomas McGrath*, 'Elizabeth Murphy, ${ }^{1}$ Thara Persaud, ${ }^{2}$ Jane Pears, ${ }^{1}$ Claire Purcell. ${ }^{1}$ Tallaght University Hospital, Dublin, Ireland; ' ${ }^{2}$ Uur Lady's Children's Hospital, Crumlin, Dublin, Ireland

\subsection{6/archdischild-2019-epa.827}

Introduction Malignant Mesothelioma (MM) is a rare invasive neoplasm arising from the mesothelial lining of several organs, primarily the pleura and peritoneum ${ }^{1}$. A causal relationship with asbestos exposure is well established, however; MM can occur in the absence of well-defined risk factors and at an earlier age ${ }^{2}$.

Case Presentation A 15 year old boy, presented to the Emergency Department with a 6-week history of cough, 5 kilogram weight loss and right sided pleuritic chest pain. He had attended the ED 4 weeks prior to this with chest pain, cough, temperature and a chest infection was diagnosed. Repeat chest $\mathrm{x}$-raydemonstrated a right lower lobe consolidation with obscuration of the hemidiaphragm and a suspected pleural based opacification. Laboratory investigations showed a hypochromic microcytic anaemia, hyponatremia and raised inflammatory markers. A CT of thorax, abdomen and pelvis showed lobulated rind of pleural based soft tissue densities in the right thorax with downward invasion through the hemidiaphragm into the liver. On transfer to a tertiary Paediatric Oncology centre, A CT-guided biopsy demonstrated a malignant epithelioid neoplasm confirming a diagnosis of malignant mesothelioma with EWSR-ATF1 rearrangement. He was treated with 2 cycles of Pemetrexed and Cisplatin Chemotherapy (NCCP guideline). Follow up imaging demonstrated disease progression which coincided with clinical deterioration. NG feeding, PleuralX drain and Oxygen therapy are part of his treatment regimen. Vinorelbine monotherapy was commenced and a Palliative approach has been adopted. Currently he is stable awaiting further imaging.

Discussion MM is rare is young people. The genetic susceptibility to MM is complex and recently an EWSR1-ATF1 fusion transcript has been described in 4 younger patients without prior asbestos exposure2. The prognosis in $\mathrm{MM}$ is poor but improved survival in younger patients has been observed1. We wish to highlight this case because it is rarely encountered in a paediatric setting.

\section{P492 ELECTROCLINICAL FEATURES AND SEIZURE OUTCOME OF PEADIATRIC PATIENTS WITH IMMUNE MEDIATED ENCEPHALOPATHY}

Ilayda Uslu*, Fatih Varol, Serhat Guler, Halit Cam, Sema Saltik. Istanbul University Cerrahpaşa-Cerrahpaşa Medical Faculty, Istanbul, Turkey

10.1136/archdischild-2019-epa.828

Purpose Most patients with immune mediated encephalitis (IME) suffer from seizures, but data on the seizure outcomes in the peadiatric population remains limited. This study was conducted to assess the clinical course and electroclinical features of peadiatric populations diagnosed with seizures associated with IME .

Methods Total 15 patients with IME were identified from Cerrahpaşa Medical Faculty Peadiatric Neurology Department and Intensive Care Unit from 2014 to 2018. The demographic,clinical and electrographical data of participants were recorded. Symptoms were classified as psychiatric,seizure,flu- 